\title{
Sensor Data Management for Asset Management
}

\author{
Rudra Bhanu Satpathy ${ }^{1}$, Siddth Kumar Chhajer ${ }^{2}$ \\ ${ }^{1}$ M.Tech,Electrical, Electronics and Communications Engineering, St. Peter's University Chennai. \\ ${ }^{2}$ MBA,Marketing, St. Peter's University, Chennai. \\ ${ }^{1}$ rudra@gmail.com, ${ }^{2}$ siddth2011@gmail.com
}

\begin{abstract}
Sensor ships in the physical context can minimize the cost of asset management and help them to enhance the lifespan of the usable product. Assessments are generally done informally in the physical surroundings and the impacts are remotely evaluated. The modeling of compromises from various origins, including governing equations, architectural exaggerations, and statistical presumptions, creates significant systemic uncertainties that change inter-point mean values. Municipal intelligent building organizations, which handle large-scale, are continually turning to data to drive their decision-making processes in asset management. The Internet of Things (IoT) enables data to be produced that can provide extra information about the present and forecast status of the infrastructure and can contribute to automating the asset management procedure.
\end{abstract}

Keywords - Sensor data, Asset management, Internet of Things

\section{Introduction}

Sensor data is the result of the technology that detects particular inputs from the physical environment and responds to them. The resulting information or input may be employed to offer knowledge or to lead a program. Sensor data management can minimize asset maintenance costs and help to increase the usable life of the system. Measuring is generally carried out indirectly in the built environment, and their causes are measured remotely. The modeling of assumptions from various sources, including boundary conditions, architectural approximations, and numeric presumptions, creates significant systematic uncertainties that change inter-point correlations. In addition, pre-construction conservatism simple guidelines that have been used correctly throughout the design phase are usually insufficient when measuring actual behavior is explained. This article discusses the distinct sensor circumstances in asset management. While this study contains comparisons with the various techniques currently suggested, little effort was made to give a comprehensive assessment of all asset management in data interpretation.

This research article aims to evaluate the role of sensor data management for asset management.

Objectives: To understand the concept of sensor data management and its benefits. To evaluate the concept of asset management and its benefit on the performance of an organization

\section{The concept of sensor data management}

As per the words of Morais et al. (2019), a sensor is an artificial execution of so-called biological meaning. A technology monitors the surroundings via a sensor and collects data. Proactively manage and transform a physical quantity into a signal. In addition, the real-world measurements are translated into digital domain data through sensors. As per the words of Potdar et al. (2018), Sensor data is the result of the technology that recognizes certain inputs from the external surroundings and responds to them. The discovered knowledge or information can be employed to reveal advice or perhaps to operate a program. On the other hand, a large variety of measurement factors are available, such as relocation, shifting, mobility, the wavelength of noise, warmth, gravity, moisture, the polarity of the electricity level, pictures, colors, and chemical characteristics. According to the words of Kaur et al. (2018), the objective of sensor data management is to identify the environmental occurrences or alterations. Alongside similar technologies, a sensor has always been employed as basic as light or as sophisticated as a supercomputer. 


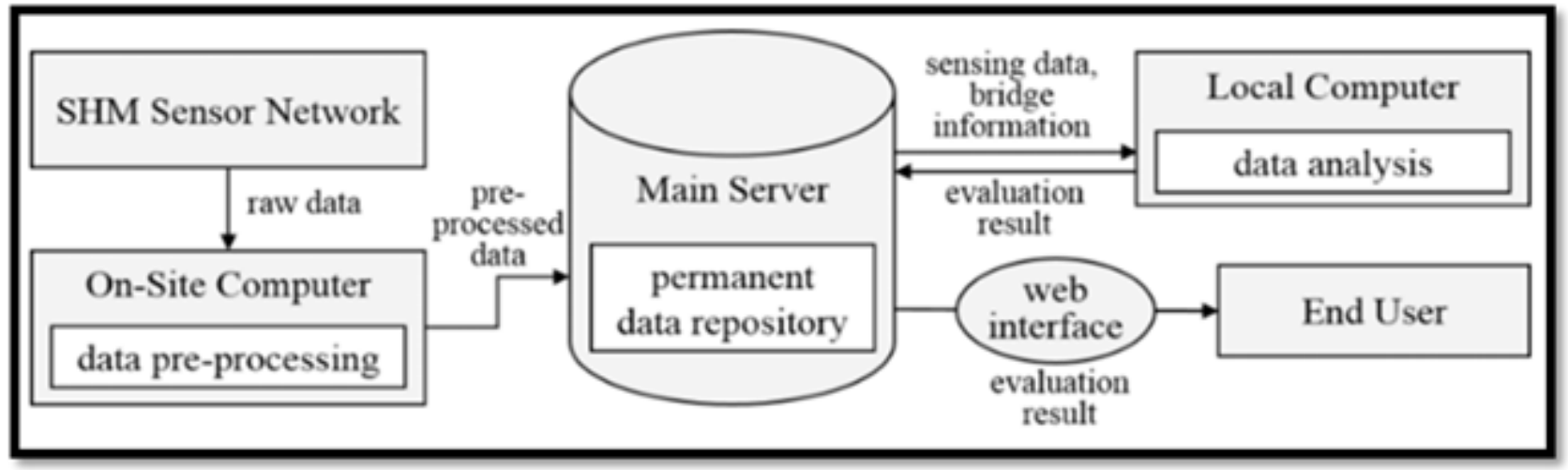

Figure 1: Sensor data management

(Source: Morais et al. 2019)

The objective of the data controller is to distinguish the logical view such as identification, accessibility, and function from the physical representation of the data in sensing devices. Consumers and applications do not need to worry about the sensor node specified, since the data sensor management provides a safer and secure database. On the other hand, considering the perspective of data management, the data management system of a network of sensors may be regarded as a decentralized information approach, although it differs from the conventional system. The sensor network data management system includes managing observable information from the examined region and responds to the user or program inquiries (Daki et al. 2017). However, while considering the rapid growth of the usage of data sensor management it is estimated that there will be more than 50 billion sensors connected to one another by the end of 2021 through the Internet of Things (IoT).

\section{The concept of asset management and its benefit}

Asset management relates to the responsibility of others to the administration of resources. As per the words of Parlikad (2019), the procedure has essentially a twin purpose - an assessment over time of the assets of a customer and risk mitigation. The expenditure is minimal, so that high net worth people, government bodies, companies, and financial intermediaries may typically benefit from this service. The standards of asset management should affect the investment management processes and plans of a company directly. On the other hand, an asset manager's duty is to determine what transaction a customer's investment will undertake or abstain from (Lee, 2021). Asset management is the process through which organizational assets are acquired, operated, maintained, renovated, and disposed of. This approach enhances the asset delivery potential and reduces costs and hazards. Effective management and correct use of technologies, processes, and people will guarantee that the capital improves positively throughout the extended enterprise.

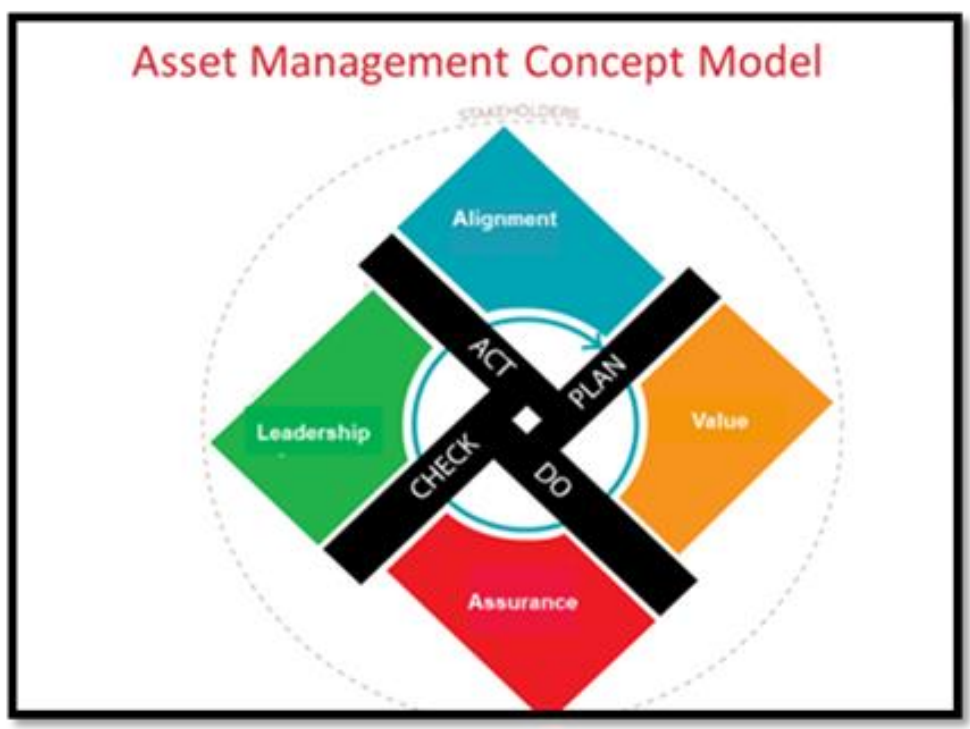

Figure 2: Conceptual model of asset management

(Source: Lee, 2021) 
Integrating Researchers to Incubate Innovation

\section{Benefits of asset management}

Smart companies don't waste time manually trying to accomplish foundation and repetitive asset management activities by repeatedly employing the same old procedure. Based on investment in asset management software, companies can automate long-term and laborious asset management operations by giving an administrative team that concentrates more on valuation activities (Jung and Kim, 2021). Here in this part of the research study, a brief overview will be provided on the benefits of asset management.

\section{Asset tracing in real-time}

Assets are a company's live component since they can increase, arrive and depart and move all around the workspace continually. This makes the tracking of assets in real-time an important element of asset management.

\section{Asset management based on the cloud}

A cloud-based solution may provide all company employees with a choice of fantastic benefits. Everyone in the organization, at a certain time and wherever, can view the full asset catalog through the utilization of asset management.

\section{Support the organization to gain complete accuracy}

Know whether a property has been lost or stolen, if it is required to be prepared and even provide accuracy and completeness. Regardless of the size of the company, tracking all the asset data and history cannot be difficult with the adoption of asset management systems.

\section{Enhance the operational efficiency of an organization}

Business industries can begin to grasp the function of each property in each sector in the firm when an asset management system is in place. This covers their capacities, their way of operation most efficiently, a comprehensive ROI, and even whether they are willing to save money.

\section{Methods and materials}

The study builds on positive philosophy and analyses previous materials such as articles and reviewed journals on the usage of sensor data for asset management via positivism research philosophy. In the development of research hypotheses and objectives, the deductive approach of this research is significant by deducting research theories and background studies (Mahmudin et al. 2020). On the other hand, the research study focuses on the application of sensor data management in the representation of an actual object, process, and service. The study uses a secondary data collection procedure which enables the researchers to get trend-based patterns from papers and journals related to data management in sensors (Sylvia, 2018). On the other hand, secondary data analysis involves a review of existing literature in order to establish the image of the topic being considered in the research study. This research study also contains a secondary data analysis that identifies and analyzes the themes that are important for the study's objective. The study piece also contains fragile data from peer-review journals, publications, and articles from 2017 through 2021 since the research study follows a secondary source of data gathering.

\section{Result and discussion}

\section{The context of the data interpretation of sensors in a constructed environment for asset management}

The context for the use of sensors in asset management in the built-in environment is described in Figure 3. This context is defined by three longitudinal axes: closeness of causation measurements, detail of the theoretical framework of the physical principle, and extent of ambiguity. In the architectural design, it is not typically difficult to evaluate all physiological features of the data directly in all directions on every element, despite enormous progress in sensor technology (Reuland et al. 2017). For the sake of determining causes, indirect measures are employed with model physical principles. 


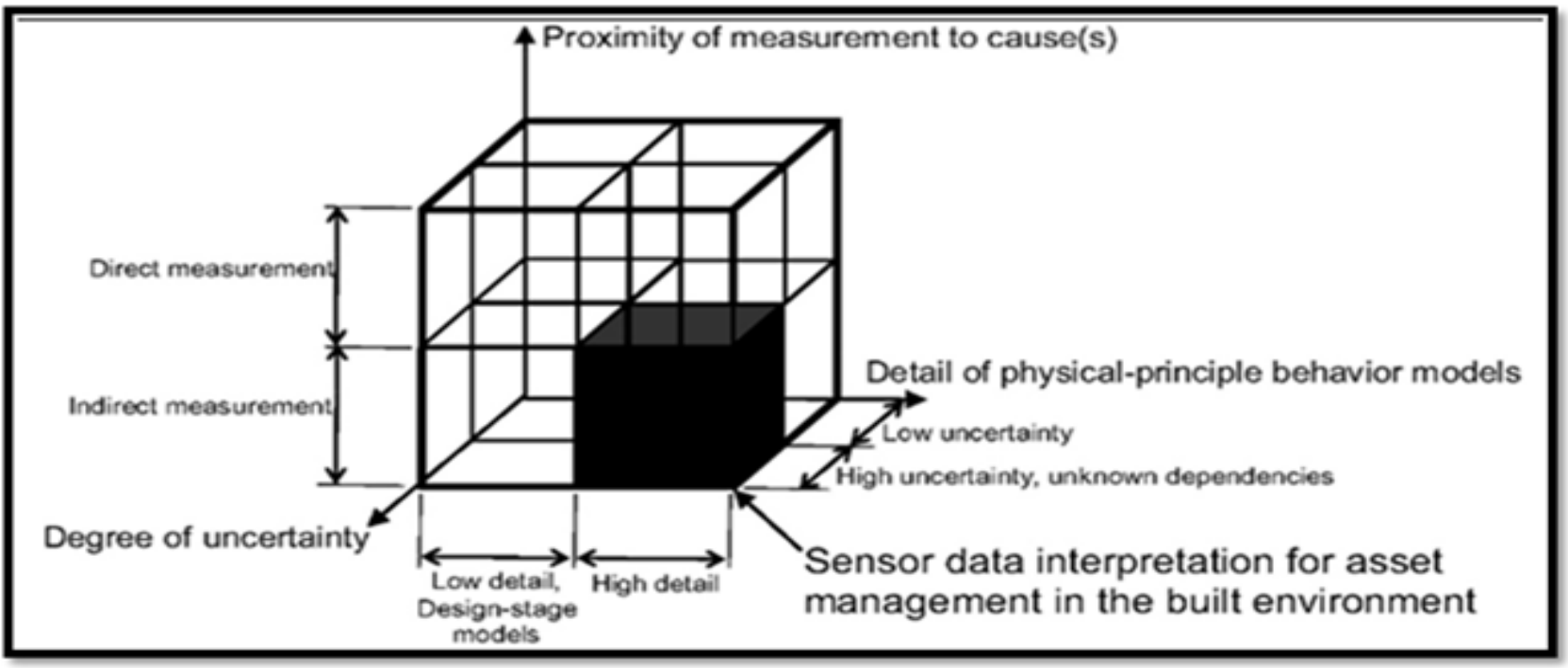

Figure 3: sensor data management for asset management

(Source: Reuland et al. 2017)

Asset management derives greatly from highly detailed, measurable, tangible behavioral models. Despite the conceptual design models generally need not be complicated, the assessment of reserve ability demands improved techniques to model elements such as degradation, actual bearing circumstances, and as-built geometry when a building is completed. As mentioned previously, improved models contribute to improving choice-taking evaluations, such as repair, upgrade, development and substitution. A lot of research was done on model-free, often termed output-only and data-driven techniques approaches used for data interpretation that was mainly designed for settings such as electrical and computer-based signal analysis (Lu et al. 2019). Although these approaches may be of relevance in some situations for harm detection, following strategic planning has limited support. Impairment is often seen by the assessors before prototype identification, and assistance to be viewed differently by different positive and negative effects is often insufficient. A low-cost alternative may be the optimum environment for model-free approaches for particularly high constructions where behavioral abnormalities are detected significantly politically as well as socially.

This is not true for most environments constructed, which is why figure 3 has this sort of application outside the sub-cube. The ecosystem created includes several levels of variation linked to observations, cost function, and model discrepancies. Ambiguities in modeling are far more frequent than uncertainties in measurement. Several of these variables cannot be represented at measuring sites using Geometric distribution. Numerous sources cause considerable systematic uncertainty since one typical feature of every network is that projected quantities are linked to values in other places at a particular location. Correlations are most subtly reliant on the extent of systemic instability. These factors provide a challenging setting for standard stochastic principles to be used in the asset management system. This study discusses further the implications of such a situation. This last distinction, shown in the "unpredictability intensity" alignment in Figure, 3 is an essential aspect in the selection of effective data interpreting techniques for the management of assets in the physical environment.

\section{Processing and management of data level sensors}

The data sensor merging process needs to take into consideration the limitations in battery life and capacity as well as the range of communications in wireless sensors for acquisition and measurement transmissions (Davila Delgado et al. 2017). These restrictions might influence the processing and administration of data at sensor nodes. Generally, autonomous vehicles require less rechargeable battery than long, sustainable sensor history to complete on-board data processing, therefore, decreasing the quantity of data to send. This means that on-board data processing is cheaper in terms of battery power saving than network link. This is crucial especially for deployments in civil constructions, in which long-term communication usually entails a specific rate and skipping the WLAN transponder uses substantial bandwidth. In order to maintain the life of mobile batteries and with the wireless sensor node onboard processing technique might be used. These methods involve engineering technology examines and noise removal on the sensor node immediately before data is sent. This section briefly examines the requirements of local data processing and data transfer with the aid of a wireless sensing node built. 


\section{Conclusion}

This research article is based on the utilization of data sensor management in the process of asset management. This research article has successfully elaborated the concept and the benefits of data sensor management, as well as the research, has also provided a sustainable viewpoint about the concept and benefits of asset management. Moreover, the research article has elaborated on the context of the data interpretation of sensors in a constructed environment for asset management. If monitoring data is properly understood, private equity is more quantitative and efficient for the constructed environment than present practices. Inhabitant's methods, such as EDMF, can explicitly reflect reasonable modeling and experimental errors for credible predictions of the generally assumed asset ages. As unknown reserve capacity may be disclosed beforehand, substantial cuts in expenditure like replacement preventatives can also be made. Future sensors will enhance their contribution to sustainable development in the constructed environment as more aged structures are rehabilitated, upgraded, and stretched than under present substitution methods.

\section{Reference}

1. Daki, H., El Hannani, A., Aqqal, A., Haidine, A. and Dahbi, A., 2017. Big Data management in smart grid: concepts, requirements and implementation. Journal of Big Data, 4(1), pp.1-19.

2. Davila Delgado, J.M., Butler, L.J., Gibbons, N., Brilakis, I., Elshafie, M.Z. and Middleton, C., 2017, September. Management of structural monitoring data of bridges using BIM. In Proceedings of the Institution of Civil Engineers-Bridge Engineering (Vol. 170, No. 3, pp. 204-218). Thomas Telford Ltd.

3. Jung, H. and Kim, B., 2021. Identifying Research Topics and Trends in Asset Management for Sustainable Use: A Topic Modeling Approach. Sustainability, 13(9), p.4792.

4. Kaur, D., Aujla, G.S., Kumar, N., Zomaya, A.Y., Perera, C. and Ranjan, R., 2018. Tensor-based big data management scheme for dimensionality reduction problem in smart grid systems: SDN perspective. IEEE Transactions on Knowledge and Data Engineering, 30(10), pp.1985-1998.

5. Lee, U.K., 2021. The Proposal of Asset Management Indicators for Public Rental Apartment. Journal of the Korea Institute of Building Construction, 21(3), pp.221-229.

6. Lu, Q., Xie, X., Heaton, J., Parlikad, A.K. and Schooling, J., 2019, October. From BIM towards digital twin: strategy and future development for smart asset management. In International Workshop on Service Orientation in Holonic and Multi-Agent Manufacturing (pp. 392-404). Springer, Cham.

7. Mahmudin, C., Sumarmo, U. and Kustiana, A., 2020. The Effect of Inductive-Deductive Approach on Students' Mathematical Creative Thinking Ability and Self-Efficacy. (JIML) JOURNAL OF INNOVATIVE MATHEMATICS LEARNING, 3(4), pp.215-226.

8. Morais, R., Silva, N., Mendes, J., Adão, T., Pádua, L., López-Riquelme, J.A., Pavón-Pulido, N., Sousa, J.J. and Peres, E., 2019. Mysense: A comprehensive data management environment to improve precision agriculture practices. Computers and Electronics in Agriculture, 162, pp.882-894.

9. Parlikad, A.K., 2019. A review of asset management literature on multi-asset systems. Reliability Engineering \& System Safety, 181, pp.181-201.

10. Potdar, V., Chandan, A., Batool, S. and Patel, N., 2018. Big energy data management for smart grids-Issues, challenges and recent developments. In Smart Cities (pp. 177-205). Springer, Cham.

11. Reuland, Y., Lestuzzi, P. and Smith, I.F., 2017. Data-interpretation methodologies for non-linear earthquake response predictions of damaged structures. Frontiers in Built Environment, 3, p.43.

12. Sylvia, M.L., 2018. Secondary data collection. Critical Analytics and Data Management for the DNP, p.61. 